\title{
Marketing social, indicadores de bienestar y ODS. Análisis de la cuenta oficial del gobierno de España @desdelamoncloa
}

\section{Social Marketing, welfare indicators and SDG. Analysis of the official account of the Spanish Government @desdelamoncloa}

Dra. Araceli Galiano Coronil es profesora e investigadora de la Universidad de Cádiz (España) (Araceli.galiano@uca.es) (https:// orcid.org/0000-0003-2270-0924)

Dra. Manuela Ortega Gil es profesora e investigadora de la Universidad de Cádiz (España) (manuela.ortega@uca.es) (https:// orcid.org/0000-0001-7013-4133)

\begin{abstract}
Resumen
Las redes sociales son instrumentos de comunicación que facilitan la sensibilización y concienciación de los ciudadanos ante problemas sociales. A través de Twitter, los gobiernos pueden obtener información útil y tomar de decisiones para mejorar el bienestar de la comunidad y concienciar a los ciudadanos sobre el logro de los Objetivos de Desarrollo Sostenible (ODS). Este trabajo muestra cómo Twitter y el marketing social sirven como herramienta en la política económica y pretende conocer si en los mensajes publicados por la cuenta oficial del gobierno de España (@ desdelamoncloa) se hablan sobre los ODS y el bienestar, además analiza el impacto de las publicaciones con contenido sobre dichos objetivos. Para ello se utiliza la metodología del análisis de contenido desde un doble enfoque, cualitativo y cuantitativo, examinando de forma descriptiva los mensajes publicados por @desdelamoncloa durante 2018, así como su relación con los ODS y el Indicador de Bienestar Sostenible. Los resultados reflejan que los ODS más comentados (los relacionados con la igualdad de género y las desigualdades) no son los que han obtenido un mayor impacto del público (trabajo y crecimiento económico), destacando los ODS incluidos en el elemento del Indicador de Bienestar Sostenible denominado «Bienestar, Economía y Sostenibilidad». Asimismo, el análisis cualitativo refleja que los mensajes publicados son de tipo informativo sobre actuaciones del gobierno y que el término «bienestar» aparece en un contexto donde se resalta los valores que representa España.
\end{abstract}

\begin{abstract}
Social media are communication tools that facilitate citizens' awareness of social problems. Through Twitter, governments can obtain useful information and make decisions to improve the well-being of the community and raise public awareness about how to achieve the Sustainable Development Goals (SDG). This work shows how Twitter and social marketing serve as economic policy instruments and aims to know if the messages published by the official twitter account of the Spanish Government (@desdelamoncloa) talk about the SDG and well-being, as well as to analyze the impact of publications with content on these objectives. The methodology of content analysis is used from a double approach, qualitative and quantitative, examining the messages published by @desdelamoncloa during 2018, as well as its relationship with the SDG and the Sustainable Wellbeing Indicator. The results reflect that the most commented SDG (those related to gender equality and inequalities) are not the ones that have obtained the greatest impact from the public (work and economic growth. Thus far, the highly valued posts have content about the SDG included in the element of the Indicator of Sustainable Welfare called Welfare, Economy and Sustainability. Likewise, the qualitative analysis reflects that the messages published are informative with content about government actions and also, the word welfare appears in a context where the values that Spain represents stands out.
\end{abstract}

\section{Palabras clave I keywords}

Marketing, social, indicadores, bienestar, desarrollo, sostenible, Twitter, gobierno.

Marketing, Social, indicators, wellbeing, development, sustainable, Twitter, government.

Cómo citar: Galiano Coronil, A., y Ortega Gil, M. (2019). Marketing social, indicadores de bienestar y ODS. Análisis de @desdelamoncloa. Retos Revista de Ciencias de la Administración y Economía, 9(18), 219-238. https://doi.org/10.17163/ret.n18.2019.03 


\section{Introducción}

Las redes sociales digitales (RSD) son un universo con una gran cantidad de información sobre preferencias, gustos, inquietudes, sensibilidades, aficiones, etc., de sus usuarios. Con ellas se han abierto nuevas oportunidades para las organizaciones, las empresas y los gobiernos para sensibilizar y concienciar a los ciudadanos ante los problemas y necesidades de la sociedad actual. De la misma forma, pueden influir en las decisiones de los usuarios aplicando técnicas de marketing social, ya que mediante esta disciplina se pretende influir en el cambio voluntario del comportamiento del público objetivo, persiguiendo mejorar su bienestar individual y el de la sociedad (Andreasen, 1994).

Desde la aparición de las RSD, el paradigma del marketing social ha sufrido un cambio, y actualmente los esfuerzos se centran en lograr que los usuarios se conviertan en promotores vocales de las causas sociales (Hestres, 2014). En relación con estas causas es importante señalar la nueva agenda internacional -Agenda 2030-, que desgrana los objetivos de la comunidad internacional en el periodo 2016-2030 para erradicar la pobreza y favorecer un desarrollo sostenible e igualitario. La Agenda 2030 gira en torno a cinco ejes centrales: Planeta, personas, prosperidad, paz y alianzas. Está integrada por 169 metas y 17 Objetivos de Desarrollo Sostenible (ODS); entre ellos se encuentra el número 3, que se refiere a la salud y el bienestar.

Diversos estudios han investigado el bienestar y los Objetivos de Desarrollo Sostenible desde varios enfoques. Froding et al. (2007) analizaron los aspectos que influyen en una ciudad saludable y sostenible, otros como los Asheim (2010); Durana et al. (2015); Costanza et al. (2016); Mikulcic, Klemes y Duic (2016); Momete (2017) y Di Maria (2019), estudiaron la relación entre bienestar y desarrollo sostenible. Costanza et al. (2016) relacionan los ODS con un nuevo indicador de bienestar, el «SWI», compuesto de tres elementos: Contribución Económica Neta, Capital Natural y Capital Social. El primero representa lo económico, el segundo la sostenibilidad y el medioambiente y el tercero el bienestar.

Sin embargo, son escasos los estudios que investigan la efectividad del bienestar y el desarrollo sostenible desde el punto de vista del marketing social en medios sociales como Twitter. Entre ellos encontramos el trabajo de Menéndez, Saura y Álvarez (2018), en el que se identifican los factores y sentimientos relacionados con el desarrollo sostenible del medio ambiente y la salud pública mediante el análisis del hashtag \#WorldEnvironmentDay. Otro enfoque es el de Sao y Lee (2018), quienes analizaron la relación entre el gobierno y el bienestar social mediante la metodología del Análisis de Redes Sociales en Twitter.

Por ello, el presente trabajo pretende avanzar en las líneas sobre felicidad y economía del bienestar, integrando el marketing social, la economía del bienestar y la política económica. Concretamente, este trabajo muestra cómo la utilización de Twitter, desde un enfoque de marketing social, es un instrumento para avanzar en el estudio del bienestar y de las políticas públicas relacionadas con el cumplimiento de los ODS. Con esta finalidad, los objetivos que se plantean son: conocer si en los mensajes publicados por @desdelamoncloa se habla sobre los ODS y bienestar, así como 
analizar el impacto de las publicaciones que tengan contenido sobre dichos objetivos y sobre el Indicador de Bienestar Sostenible.

Para ello se han analizado los 2.587 tweets de la cuenta oficial del Gobierno de España (@desdelamoncloa) publicados durante 2018, utilizándose softwares de análisis de datos IBM SPSS y Nvivo 12 plus. La metodología utilizada ha sido el análisis de contenido, desde un enfoque cualitativo y cuantitativo, mediante la que se han codificado los tweets de acuerdo con los Objetivos de Desarrollo Sostenible (ODS), identificando, por un lado, los más valorados por los usuarios de Twitter, y por otro lado los términos más utilizados por la cuenta de la organización objeto de estudio. Asimismo, se ha planteado la hipótesis de que el impacto de los mensajes publicados (medidos por la suma de los likes+retweets) por @desdelamoncloa depende de los ODS que en ellos se traten.

\section{Marco teórico}

Desde que en el año 1952 Wiebe se preguntó ¿Por qué no se vende la solidaridad como se vende el jabón?, la cuestión sobre la poca efectividad de los vendedores de las causas sociales ha sido estudiada a lo largo del tiempo, dando lugar a diversos matices del denominado «marketing social». El punto de partida se sitúa en 1969 cuando Kotler y Levy (1969a) sugieren una nueva dimensión del marketing que supone una extensión al campo de las ideas y de las organizaciones sin ánimo de lucro como las iglesias, las escuelas públicas o las administraciones, puesto que también ellas poseen productos que ofrecer a los clientes. Por tanto, se evidencia que la "esencia del marketing reside en una idea general de intercambio de valor más que en la reducida tesis de las transacciones del mercado" (Kotler \& Levy, 1969b, p. 57).

Más adelante, Andreasen (1995) añadió que el «marketing social» ha de tener la capacidad de modificar los comportamientos en busca de un bien superior (individual y social), siendo dicho cambio de actitud o comportamiento, de forma voluntaria, lo que ha caracterizado a esta disciplina en los últimos años.

Sin embargo, el paradigma del «marketing social» ha evolucionado desde la aparición y uso de las redes sociales como herramientas de comunicación. Desde esta perspectiva, Hestres (2014) planteó una estrategia que cambió la visión del social marketing. Ésta consiste en pensar que, en lugar de gastar la mayor parte de los recursos en la educación, es en el cambio de actitud y de comportamiento donde las organizaciones deberían focalizar sus esfuerzos para lograr comprender el uso de las RSD. Esto dará la posibilidad de ofrecer al público contenido interesante y, por tanto, una mayor difusión y visibilidad de la información. De este modo, las organizaciones podrán trabajar en determinar cómo transformar a sus partidarios en promotores vocales de sus causas (Kotler, 2011; Dooley et al., 2012; Bernhardt, Mays \& Hall, 2012).

En este sentido Twitter se ha configurado como una herramienta idónea, al conformar un espacio público online donde las organizaciones pueden informar y recibir feedback de sus aportaciones, ya que es una plataforma interactiva en la que los usuarios y las organizaciones pueden compartir información. Diversos estudios avalan su idoneidad como herramienta de comunicación en la esfera pública y en la política como Golbeck y Hansen (2014); McGregor y Mourao (2016); Bain y Chaban 
(2017), entre otros. Recientemente muchas investigaciones se han dirigido a temas relacionados con la salud (Chen et al. 2017; Pershad et al., 2018; Aboelmaged, 2018; Zhang \& Ahmed, 2019; Colditz et al., 2019; Chua et al., 2019).

Varios investigadores han centrado sus estudios en las relaciones entre felicidad, estado del bienestar y políticas públicas (Gerdtham \& Johannesson, 2001; Easterlin, 2010; Di Tella, Haisken \& MacCulloch, 2010), mientras que se han buscado indicadores que midan la felicidad o el bienestar de los individuos, los ciudadanos y las empresas. Otros se han centrado en la felicidad desde el punto de vista empresarial (Fisher, 2010; Blanchflower \& Oswald, 2011; Ravina, Villena \& Gutiérrez, 2017; Frey \& Stutzer, 2018), principalmente para asignar de forma racional los recursos escasos entre sus diferentes alternativas, distribuir los insumos y/o factores productivos de forma más eficiente y repartir más equitativamente los bienes y/o servicios entre las comunidades. La teoría del bienestar en forma racional conduce a minimizar los niveles de desigualdad social, y a establecer una distribución de los recursos más eficiente (Duarte \& Jiménez, 2007).

Desde finales del siglo pasado se han desarrollado numerosos indicadores que miden el desarrollo y el bienestar. Entre los más utilizados se encuentran el Índice para una vida mejor (BLI, por su acrónimo en inglés), Índice de Desarrollo Humano (IDH), el Índice de Pobreza Humana (IPH), las Necesidades Básicas Insatisfechas (NBI), el Índice de Desarrollo Sostenible (IDS), Índice Progreso Real (IPG) y el Índice de Bienestar Sostenible (SWI, por su acrónimo en inglés).

El deterioro del medio ambiente y sus efectos están provocando que tanto las investigaciones como las políticas pongan el foco en este problema. El Informe Stiglitz-Sen-Fitoussi (2008) analiza el bienestar y distingue entre la evaluación del bienestar actual y su sostenibilidad. Al bienestar actual se considera pluridimensional y depende de factores económicos -recursos- y no económicos -lo que los ciudadanos hacen y pueden hacer, la forma en que perciben sus vidas y su entorno natural-. La sostenibilidad del bienestar actual pretende que se transmitan a las generaciones futuras los stocks que son importantes para nuestras vidas -capital físico, humano, social y natural-. Además, considerará que el sistema estadístico se debe centrar más en medir el bienestar de sus ciudadanos en un contexto de sustentabilidad que en la medición de la producción económica y propone 12 recomendaciones para mejorar las estadísticas sobre el bienestar de la población; cinco sobre el bienestar material, cinco referentes a la calidad de vida y dos la sostenibilidad del medioambiente.

Según el informe Brundtland Nuestro Futuro Común, el desarrollo sostenible es aquél que "satisface las necesidades del presente sin comprometer la capacidad de las generaciones futuras de satisfacer las propias” (Brundtland, 1987, p. 23). En el año 2000, la Organización de Naciones Unidas proclamó los ocho Objetivos de Desarrollo del Milenio (ODM): Erradicar la pobreza extrema y el hambre (1), lograr la enseñanza primaria universal (2), promover la igualdad entre los géneros y la autonomía de la mujer (3), reducir la mortalidad infantil (4), mejorar la salud materna (5), combatir el VIH/SIDA, el paludismo y otras enfermedades (6), garantizar la sostenibilidad del medio ambiente (7) y fomentar una asociación mundial para el desarrollo (8). Posteriormente, en la Conferencia de Río de Janeiro de Naciones Unidas sobre Desarrollo Sostenible (RIO+20) del año 2012 se elaboraron 17 Objetivos de Desarrollo 
Sostenible (ODS) con objeto de poner fin a la pobreza, proteger el planeta y garantizar la paz y prosperidad de las personas (ver Tabla 1).

Estos objetivos son una llamada universal a la adopción de medidas para poner fin a la pobreza, proteger el planeta y garantizar que todas las personas gocen de paz y prosperidad e incluyen nuevas esferas como el cambio climático, la desigualdad económica, la innovación, el consumo sostenible y la paz y la justicia, entre otros. Se centran en el bienestar, en un avance económico responsable y en la protección del medio ambiente. Cada día más autores sostienen que el desarrollo debe estar enfocado al bienestar y a la sostenibilidad (Coulthard, Johnson \& McGregor, 2011; Rogers et al., 2012; Costanza et al., 2016; Rodrigo-Cano, Picó \& Dimuro, 2019). Según Rogers et al. (2012) la sostenibilidad social y ambiental requieren un enfoque basado en el bienestar y en la satisfacción de las necesidades humanas. Para la presente investigación destacamos el objetivo 3, cuyo fundamento es garantizar una vida saludable y promover un estado de bienestar.

Para medir el bienestar social se han desarrollado diversos indicadores como el PIB corregido, el Índice de Desarrollo Humano o el Índice de Planeta Feliz que miden aspectos anteriormente mencionados, con la finalidad de crear políticas que ayuden a incrementar la calidad de vida de los ciudadanos. Sin embargo, existe poca literatura que estudie el bienestar y el desarrollo sostenible en las redes sociales, concretamente en Twitter (v. gr. Fownes, Yu \& Margolin, 2018; Pearce, 2014).

Costanza et al. (2016) han elaborado métodos para relacionar los ODS a las medidas globales de bienestar sostenible. Para ellos, en un mundo interconectado, los ODS no se pueden lograr a menos que haya un bienestar sostenible a escala mundial. Se basan en la idea de que el mejor sistema es aquel cuyo objetivo general se basa en una vida de alta calidad, próspera y equitativa de sus ciudadanos que, a su vez, sea sostenible. En este sentido proponen un nuevo indicador que sea capaz de medir el bienestar y que integre los conocimientos actuales de la ecología, la economía, la psicología y la sociología para ello. Este indicador es el «SWI» (Sustainable Wellbeing Index) que es un híbrido que combina tres partes básicas para un bienestar sostenible desde una dimensión económica, pero incluyendo tanto a la sociedad como la naturaleza. Está compuesto por tres elementos: Contribución Económica Neta (E) o asignación eficiente (elemento económico), Capital Natural / Contribución a los Servicios del Ecosistema (N) o escala sostenible (elemento medioambiental) y Capital Social / Contribución Comunitaria (S) o distribución justa (elemento del bienestar). Además, en su trabajo (op. cit.) relacionan estos tres elementos que forman el indicador de bienestar sostenible (SWI) con los 17 Objetivos de Desarrollo Sostenible (ODS) (ver Tabla 1). 


\section{Tabla 1. Relación SWI y ODS. Los 17 ODS agrupados en los tres elementos del bienestar sostenible}

\begin{tabular}{|c|c|}
\hline \multicolumn{2}{|c|}{ Asignación eficiente: construyendo una economía viva (E) } \\
\hline Objetivo 7 & $\begin{array}{l}\text { Asegurar el acceso a energía asequible, confiable, sostenible y moder- } \\
\text { na para todos. }\end{array}$ \\
\hline Objetivo 8 & $\begin{array}{l}\text { Promover el crecimiento económico sostenido, inclusivo y sostenible, } \\
\text { el empleo pleno y productivo y el trabajo decente para todos. }\end{array}$ \\
\hline Objetivo 9 & $\begin{array}{l}\text { Construir infraestructura resistente, promover la industrialización } \\
\text { inclusiva y sostenible y fomentar la innovación. }\end{array}$ \\
\hline Objetivo 11 & $\begin{array}{l}\text { Hacer que las ciudades y los asentamientos humanos sean inclu- } \\
\text { sivos, seguros, resilientes y sostenibles. }\end{array}$ \\
\hline Objetivo 12 & Asegurar patrones de consumo y producción sostenibles. \\
\hline \multicolumn{2}{|c|}{ Escala sostenible: permanecer dentro de los límites planetarios (N) } \\
\hline Objetivo 6 & $\begin{array}{l}\text { Asegurar la disponibilidad y la gestión sostenible del agua y el san- } \\
\text { eamiento para todos. }\end{array}$ \\
\hline Objetivo 13 & $\begin{array}{l}\text { Tomar medidas urgentes para combatir el cambio climático y sus } \\
\text { impactos. }\end{array}$ \\
\hline Objetivo 14 & $\begin{array}{l}\text { Conservar y utilizar de manera sostenible los océanos, mares y re- } \\
\text { cursos marinos para el desarrollo sostenible. }\end{array}$ \\
\hline Objetivo 15 & $\begin{array}{l}\text { Proteger, restaurar y promover el uso sostenible de los ecosistemas } \\
\text { terrestres, gestionar de forma sostenible los bosques, combatir la } \\
\text { desertificación, detener y revertir la degradación de la tierra y de- } \\
\text { tener la pérdida de biodiversidad. }\end{array}$ \\
\hline \multicolumn{2}{|c|}{ Distribución justa: capacidades de protección para el florecimiento (S) } \\
\hline Objetivo 1 & Poner fin a la pobreza en todas sus formas en todas partes. \\
\hline Objetivo 2 & $\begin{array}{l}\text { Poner fin al hambre, lograr la seguridad alimentaria y mejorar la } \\
\text { nutrición, y promover una agricultura sostenible. }\end{array}$ \\
\hline Objetivo 3 & $\begin{array}{l}\text { Asegurar vidas saludables y promover el bienestar para todos en } \\
\text { todas las edades. }\end{array}$ \\
\hline Objetivo 4 & $\begin{array}{l}\text { Asegurar una educación de calidad inclusiva y equitativa y promov- } \\
\text { er oportunidades de aprendizaje de por vida para todos. }\end{array}$ \\
\hline Objetivo 5 & $\begin{array}{l}\text { Lograr la igualdad de género y empoderar a todas las mujeres y } \\
\text { niñas. }\end{array}$ \\
\hline Objetivo 10 & Reducir la desigualdad dentro y entre los países. \\
\hline Objetivo 16 & $\begin{array}{l}\text { Promover sociedades pacíficas e inclusivas para el desarrollo sos- } \\
\text { tenible, brindar acceso a la justicia para todos y crear instituciones } \\
\text { eficaces, responsables e inclusivas a todos los niveles. }\end{array}$ \\
\hline Objetivo 17 & $\begin{array}{l}\text { Fortalecer los medios de implementación y revitalizar la asociación } \\
\text { global para el desarrollo sostenible. }\end{array}$ \\
\hline
\end{tabular}

Fuente: Costanza et al., 2016. 


\section{Materiales y método}

Para recopilar la información a analizar se ha utilizado el análisis de contenido desde una perspectiva tanto cualitativa como cuantitativa. La primera permite verificar la presencia de temas, palabras o conceptos en un contenido; mientras que la segunda tiene como objetivo medir los datos y establecer la frecuencia de aparición de los elementos considerados como unidades de información. Krippendorf (1990) define el análisis de contenido como "una técnica de investigación destinada a formular, a partir de ciertos datos, inferencias reproducibles y válida que puedan aplicarse a su contexto" (p. 28). Esta metodología es apropiada para este trabajo ya que adopta como unidad de análisis al mensaje que se selecciona conforme a reglas explícitas (Neuendorf, 2002). El análisis se ha realizado en tres etapas siguiendo el procedimiento de Bardin (1983): identificación de las unidades de análisis, codificación de los mensajes y análisis de resultados.

En la primera etapa se han identificado las unidades de análisis en relación con los objetivos a alcanzar. Siguiendo estos pasos se han identificado como unidades de estudio los mensajes publicados por el usuario@desdelamoncloa, cuenta oficial en Twitter del Gobierno de España, durante 2018. Se ha considerado para el análisis la red de microblogging Twitter, ya que es una de las redes sociales más notorias y utilizadas en España (IAB, 2018), con unos 4,9 millones de usuarios (SMF, 2018). Además, es cada vez más útil para la extracción de información sobre la opinión pública en las redes sociales (Bernhardt, Mays y Hall, 2012).

A diferencia de Facebook, que ofrece limitaciones con perfiles privados o semiprivados, Twitter facilita la comunicación abierta entre los usuarios y les permite compartir sus opiniones de manera públicamente accesible. Para la extracción de los datos se ha utilizado la herramienta FanPageKarma. Con este recurso se han recopilado todos los mensajes emitidos en 2018 por @desdelamoncloa, reuniendo una muestra total efectiva de 2.587 publicaciones (unidades informativas).

En la segunda etapa se ha procedido a la codificación de dichos posts según tres variables: v1 objetivo (o1...o17), v2 mes (enero...diciembre) y v3 impacto (likes +compartidos). Para codificar los mensajes según la variable 1 se ha tenido en cuenta que estos contengan palabras o expresiones relacionadas con cada ODS (ver Tabla 2). Asimismo, para medir el impacto (variable 3 ) del mensaje se ha sumado las veces que estos se han compartido más los likes de cada uno.

Tabla 2. Palabras y expresiones relacionadas con los ODS (v1)

\begin{tabular}{|c|l|}
\cline { 2 - 2 } ODS & \multicolumn{1}{c|}{ Palabras } \\
\hline Objetivo 1 & Pobreza \\
\hline Objetivo 2 & Hambre, malnutrición \\
\hline Objetivo 3 & Bienestar, calidad de vida, salud, sanidad \\
\hline Objetivo 4 & Alumno, beca, educación \\
\hline Objetivo 5 & Desigualdad, género, hombres, mujeres, violencia machista \\
\hline Objetivo 6 & Agua, potable \\
\hline
\end{tabular}




\begin{tabular}{|c|l|}
\hline \multicolumn{1}{|c|}{ ODS } & \multicolumn{1}{c|}{ Palabras } \\
\hline Objetivo 7 & Energía \\
\hline Objetivo 8 & Desempleo, empleo, paro, crecimiento económico, salario \\
\hline Objetivo 9 & Desarrollo, industria, infraestructura, innovación, investigación \\
\hline Objetivo10 & Igualdad, renta \\
\hline Objetivo 11 & Ciudades, comunidades, sostenible \\
\hline Objetivo 12 & Consumo, producción \\
\hline Objetivo 13 & Cambio climático, clima, temperatura \\
\hline Objetivo 14 & Marino, océano \\
\hline Objetivo 15 & Ambiental, contaminación, fauna, flora, monte, río \\
\hline Objetivo 16 & Justicia, ONG \\
\hline Objetivo 17 & Alianza, convenio, relaciones \\
\hline
\end{tabular}

Fuente. Elaboración propia

En la tercera etapa, análisis de los resultados, se profundiza en el conocimiento de los mensajes. Los recursos utilizados para ello han sido los programas IBM SPSS y Nvivo 12 plus, que permiten realizar dos clases de análisis: el primero descriptivo, tanto cualitativo como cuantitativo para conocer: los términos más utilizados, el contexto en el que aparece la palabra bienestar, el impacto de los mensajes en los que se habla sobre los ODS y el de aquellos relacionados con el SWI. Y el segundo correlacional, de dos tipos, uno con el propósito de medir el grado de relación que existe entre el impacto de los mensajes (v3) y los ODS (v1), que se llevaría a cabo mediante la prueba no paramétrica de Kruskal Wallis en el SPSS. Y otro, mediante un análisis de conglomerados utilizando el coeficiente de correlación de Pearson en el programa Nvivo 12, visualizándose los nodos que comparten palabras similares. Además, para mostrar otros resultados, se ha recurrido a recursos como tag clouds y árboles de palabras.

\section{Resultados}

En el análisis de resultados se muestra en primer lugar una descripción general de la cuenta@desdelamoncloa. A continuación, los datos del análisis cualitativo y por último los resultados correspondientes al análisis cuantitativo. La figura 1 muestra que la cuenta oficial del Gobierno en España sigue a 154 usuarios y cuenta con 591.769 seguidores. Estos datos ofrecen una visión de la personalidad de la cuenta, medida por el ratio TFF (Twitter Following Follower Ratio) (Moll, 2015). En el caso que nos ocupa, al ser la cuenta analizada una institución pública de reconocido prestigio, el valor del ratio es muy elevado (591.769/154), lo que significa que resulta difícil transmitir una sensación de cercanía. 
Figura 1. Datos generales de Twitter de @desdelamoncloa

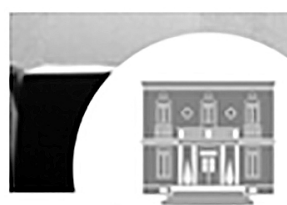

La Moncloa

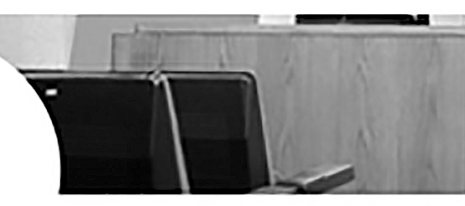

$\begin{array}{ccc}\text { Terets } & \text { Sizuienco } & \text { Seguisorts } \\ 35,7 \mathrm{mil} & 154 & 592 \mathrm{~m}\end{array}$

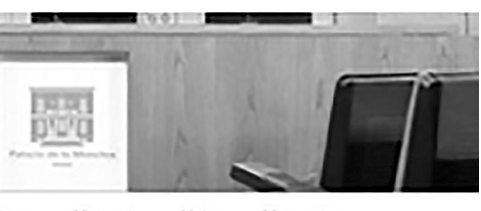

La Moncloa 0 Odesdetamondios

Bichenidos a bovents of sis on twitter del Gooitro de otsports 2

C Madrid (Souin)

8 lamoncioagobes

0 Ver transmisiones

G Seunib en juío de 2009

\section{Tweets Tweets y respuestas Multimedia}

9

Fuente: Recuperado el 26/05/19 de @desdelamoncloa https://bit.ly/2YVIrrC

Si se observa la evolución mensual de los mensajes emitidos durante 2018 y el impacto provocado en el público por dichas publicaciones (likes+retweets) se obtienen los datos de la figura 2, que muestra que el mayor impacto medio por mensaje se obtuvo en el mes de junio. En este mes, en la madrugada del sábado 9 de junio al domingo 10, se produjo el rescate por parte del buque Aquarius de 629 inmigrantes que intentaban alcanzar la costa europea, entre los que se encontraban 123 menores no acompañados, 11 niños pequeños y 7 mujeres embarazadas.

Figura 2. $N^{0}$ de mensajes emitidos por @desdelamoncloa e impacto medio por mensaje durante 2018

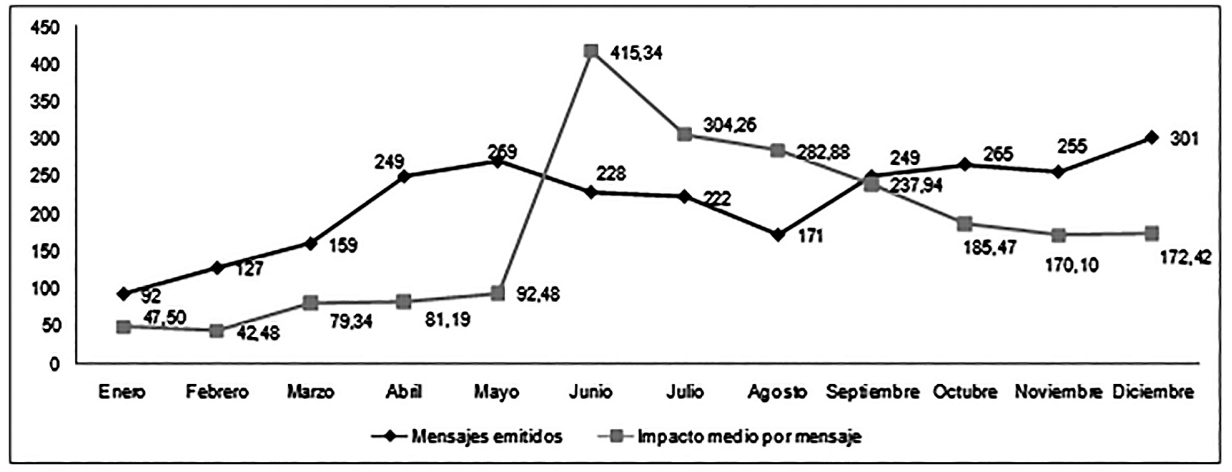

Precisamente el mensaje con más impacto en todo el año (figura 3) trata el tema de acogida del buque Aquarius, que consiguió un total de 7.201 reacciones (retweets+likes). 
Figura 3. El mensaje con mayor impacto de @desdelamoncloa en 2018

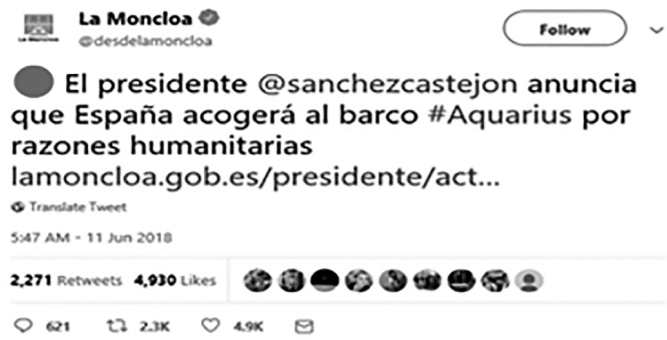

Fuente: Recuperado el 26/05/2019 de @desdelamoncloa https://bit.ly/2YPP76r

\subsection{Resultados del análisis cualitativo}

A pesar de que el mensaje con mayor reacción del público es el relacionado con el rescate del Aquarius, los términos más frecuentes en las publicaciones de junio son «rueda» y "prensa» (Figuras 4 y 5), que hacen referencia a las apariciones de miembros del gobierno ante los periodistas. Estos resultados denotan la tendencia a publicar mensajes de tipo informativo en Twitter, sobre todo de acuerdos o decisiones tomadas por el gobierno. Lo mismo ocurre el resto del año (Figura 5), en el que el protagonismo de otros términos importantes como \#agenda2030 es menor (aparece en el $13 \%$ de los mensajes).

Figura 4. Términos más publicados en junio 2018

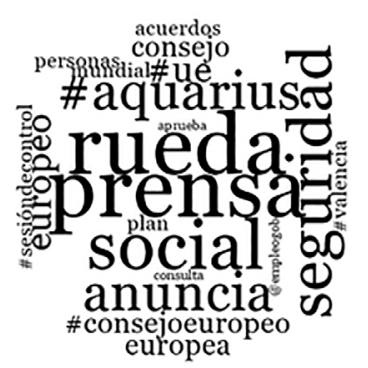

Figura 5. Términos más publicados en 2018

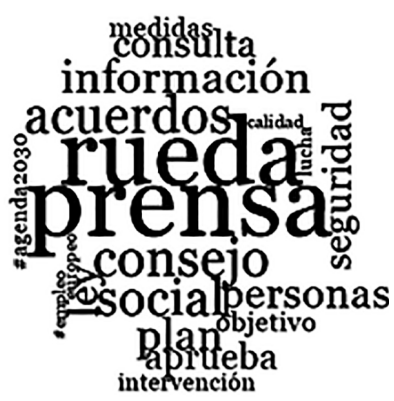

Tampoco aparecen los vocablos ODS u «Objetivos de Desarrollo Sostenible», aunque sí la palabra «objetivo», que hace referencia a programas concretos relacionados con dichos ODS, como por ejemplo el de la educación (ver Figura 6). 
Figura 6. Tweet en el que se aborda el ODS 4 «Educación de calidad»

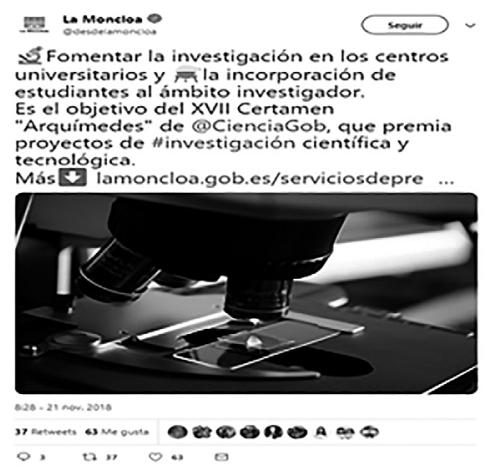

Fuente: Recuperado el 21/11/2018 de @desdelamoncloa de https://bit.ly/2YOiB8D

Haciendo especial referencia al ODS 3, "Salud y bienestar», uno de los más destacados e investigados desde un enfoque multidisciplinar, en la Figura 7 se muestra el contexto en el que aparece el término «bienestar» en la cuenta objeto de estudio. Este vocablo aparece con otras palabras como «Estado» o con los hashtags \#40AñosdeEstadoSocial o \#SomosEspaña, lo que vienen a indicar la preocupación del gobierno por los valores que representa España y por el bienestar de los ciudadanos.

Figura 7. Contexto en el que aparece el término bienestar en las publicaciones de @desdelamoncloa en 2018

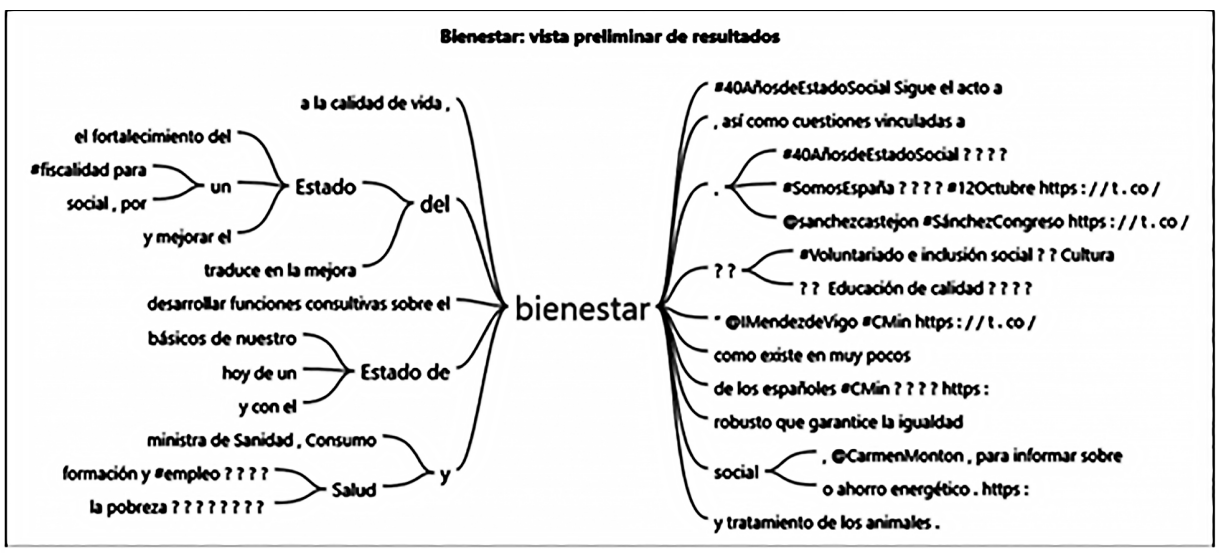

Fuente: Elaboración propia. 
Para conocer la relación del objetivo 3 (bienestar y salud) con otros ODS, se ha llevado a cabo un análisis de conglomerados (Figura 8), en el que se destaca que los mensajes que hablan de los objetivos 3, 5 (igualdad de género) y 10 (reducción de las desigualdades) son similares entre ellos. Si examinamos de forma más concreta los mensajes que hablan de estos dos últimos, destaca que tienen un impacto de 4.831 reacciones. De ello se puede deducir que a los seguidores de @desdelamoncloa les preocupa las condiciones de desigualdad y relacionan este aspecto con el bienestar.

Figura 8. Análisis de conglomerados de los mensajes sobre ODS por similitud de palabras

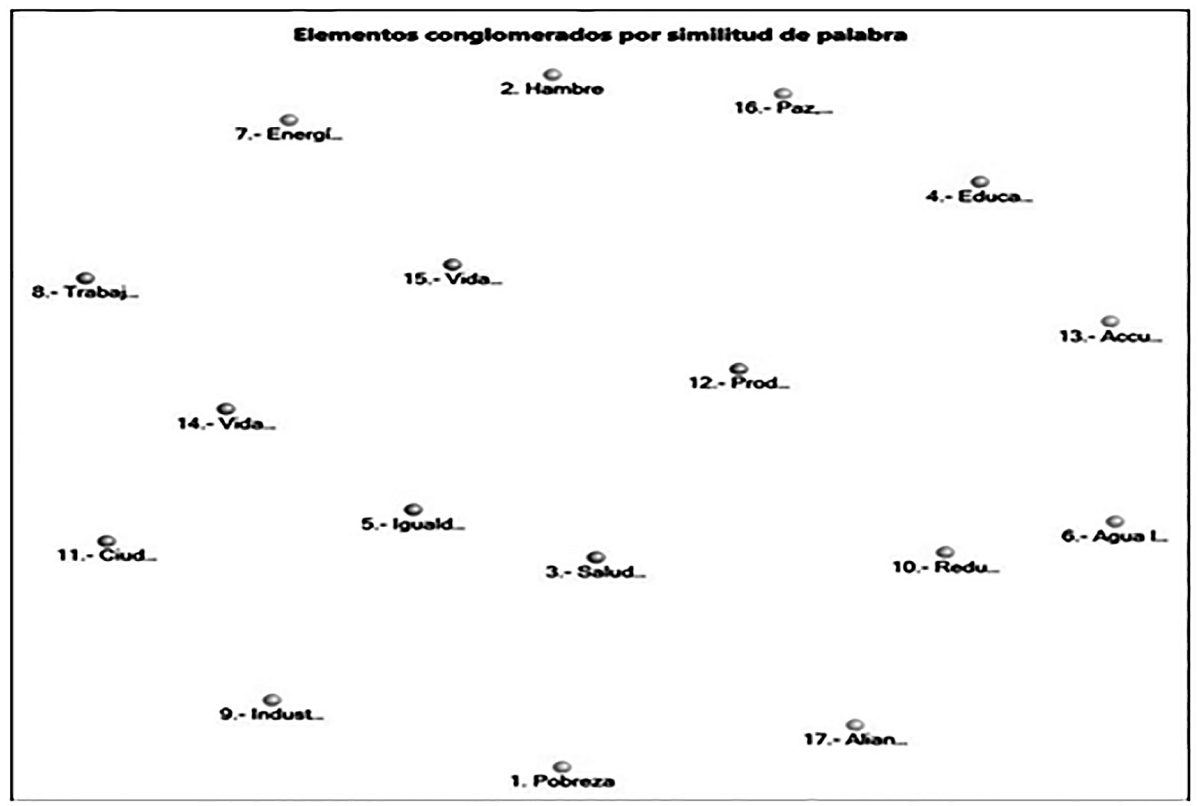

Fuente: Elaboración propia.

\subsection{Resultados del análisis cuantitativo}

Después de una primera exploración de los datos desde un enfoque cualitativo, se muestran los resultados del análisis cuantitativo. Se ha comenzado analizando el número de mensajes en los que se habla de algún o algunos ODS, así como el impacto medio de dichas publicaciones (Figura 9). 
Figura 9. Número de mensajes emitidos por ODS e impacto de los mismos en 2018 por @desdelamoncloa

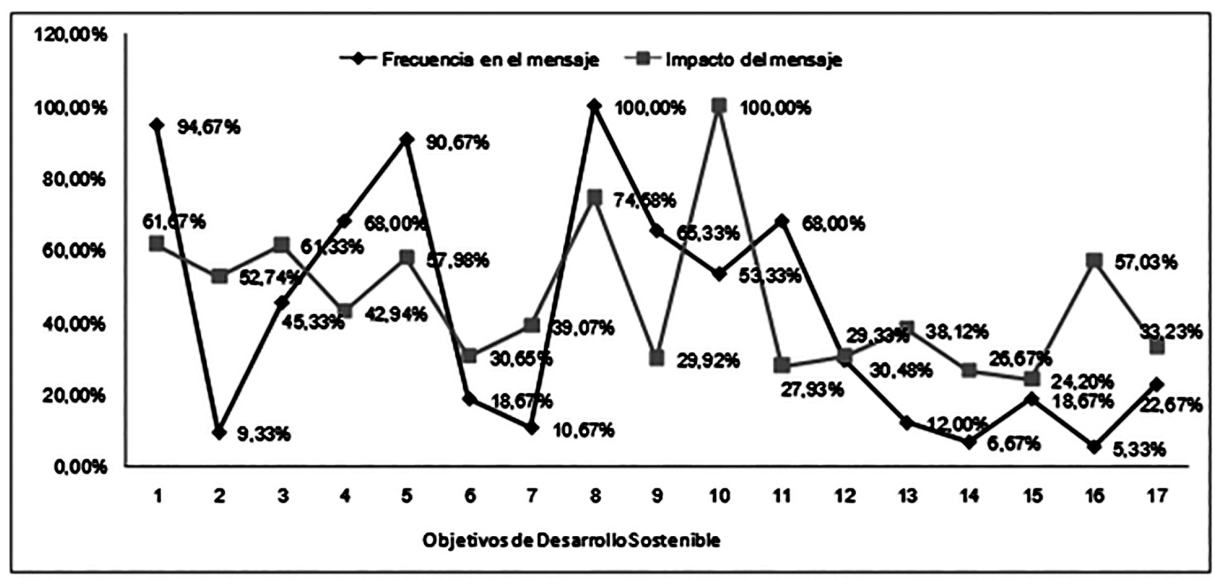

Fuente: Elaboración propia.

Como se puede comprobar en la figura ut supra reflejada, los mensajes que hablan del ODS 8, es decir, «trabajo decente y crecimiento económico» han sido los que han tenido un mayor impacto, sin embargo, no son los más publicados. Lo contrario ocurre con los mensajes que hablan del objetivo 10, «reducción de las desigualdades», que son los más publicados, pero tienen un impacto del 53.33\%. Por otra parte, los mensajes con menos impacto son los que hablan del ODS 15 y 11. En referencia al ODS de «salud y bienestar» (3) destaca que el impacto medio por mensaje es de $61.33 \%$, de lo que se deduce que es uno de los más importantes. Otros objetivos que aparecen con poca frecuencia son el 16 y el 14 .

En general, los ODS más publicados por la organización no son los que han causado una mayor reacción en el público. En este sentido, es preciso comentar que la mayor diferencia está en el objetivo 16, "paz justicia e instituciones sólidas»y en el 2, «hambre cero». En ambos la reacción del público ha sido mucho mayor en comparación con el número $(n)$ de mensajes publicados en los que se habla de ellos. Para una visión con más detalle, en la Tabla 3 se observan los mensajes que tratan varios objetivos a la vez, en concreto, se muestran aquellos mensajes que contienen más de tres.

Tabla 3. Mensajes publicados por @desdelamoncloa que contienen más de tres objetivos en 2018

\begin{tabular}{|l|l|l|l|l|}
\hline \multicolumn{1}{|c|}{ Id } & \multicolumn{1}{c|}{ Objetivo } & $\mathbf{N}^{\text {o }}$ de objetivos & \multicolumn{1}{c|}{$\mathbf{N}^{\text {o }}$ Me gusta } & \multicolumn{1}{c|}{ Retuits } \\
\hline 3 & $1,3,4,5,7,8,10,12$ & 8 & 534 & 300 \\
\hline 1346 & $1,4,5,10,11,12$ & 6 & 242 & 150 \\
\hline
\end{tabular}




\begin{tabular}{|l|l|l|l|l|}
\hline \multicolumn{1}{|c|}{ Id } & \multicolumn{1}{|c|}{ Objetivo } & $\mathbf{N}^{\text {o }}$ de objetivos & $\mathbf{N}^{\text {o Me gusta }}$ & \multicolumn{1}{c|}{ Retuits } \\
\hline 72 & $3,4,8,10$, & 4 & 2588 & 952 \\
\hline 5 & $1,5,13$ & 3 & 180 & 120 \\
\hline 66 & $1,4,9$ & 3 & 59 & 36 \\
\hline 116 & $1,5,10$ & 3 & 51 & 59 \\
\hline 397 & $1,2,5$ & 3 & 524 & 320 \\
\hline 478 & $1,4,9$ & 3 & 48 & 34 \\
\hline 479 & $5,10,13$ & 3 & 498 & 250 \\
\hline 529 & $1,4,9$ & 3 & 39 & 20 \\
\hline 803 & $4,5,10$ & 3 & 53 & 27 \\
\hline 823 & $8,9,10$ & 3 & 211 & 149 \\
\hline 1340 & $1,9,11$ & 3 & 539 & 365 \\
\hline 1423 & $9,11,16$ & 3 & 326 & 213 \\
\hline
\end{tabular}

Fuente: Elaboración propia.

Llegados a este punto se hace conveniente contrastar la hipótesis "el impacto de los mensajes publicados por @desdelamoncloa depende de los ODS que traten". Para ello se ha llevado a cabo el contraste de hipótesis no paramétrico de Kruskal Wallis, obteniéndose un p-valor inferior a 0.05 , por lo que existen diferencias significativas entre la variable impacto y la variable objetivo y puede afirmarse que el impacto de los mensajes depende del objetivo del que trate.

Tabla 4. Resultados de la prueba de Kruskal Wallis

\begin{tabular}{|l|l|}
\hline \multicolumn{2}{|c|}{ Estadísticos de prueba ${ }^{\text {a, b }}$} \\
\hline a.b & Impacto \\
\hline Chi-cuadrado & 34,622 \\
\hline gl & 16 \\
\hline Sig. asintótica &, 004 \\
\hline
\end{tabular}

Fuente: Elaboración propia.

a. Prueba de Kruskal Wallis

b. Variable de agrupación: Objetivos

Por último, en la Tabla 5 se ha examinado el impacto de los tres elementos del «SWI» según el impacto de los ODS (Costanza et al., 2016). 
Tabla 5. Impacto de los elementos del SWI según su relación con los ODS
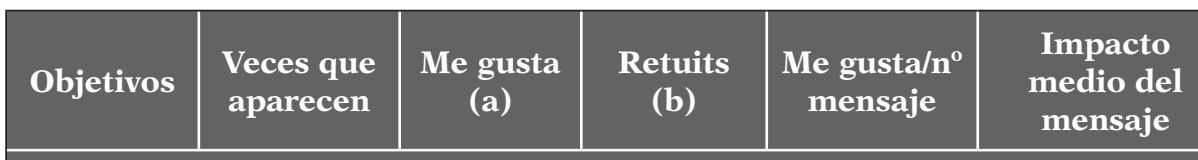

Distribución justa. Capital Social/ Contribución Comunitaria (S)

\begin{tabular}{|l|l|l|l|l|l|}
\hline 1 & 71 & 13840 & 9539 & 194,93 & 329,28 \\
\hline 2 & 7 & 1171 & 800 & 167,29 & 281,57 \\
\hline 3 & 34 & 7105 & 4028 & 208,97 & 327,44 \\
\hline 4 & 51 & 7286 & 4406 & 142,86 & 229,25 \\
\hline 5 & 68 & 12783 & 8267 & 187,99 & 309,56 \\
\hline 10 & 40 & 13268 & 8089 & 331,70 & 533,93 \\
\hline 16 & 4 & 739 & 479 & 184,75 & 304,50 \\
\hline 17 & 17 & 1889 & 1127 & 111,12 & 177,41 \\
\hline & 275 & 58081 & 36735 & 1529,60 & 2492,94 \\
\hline
\end{tabular}

Escala sostenible. Capital Natural / Contribución a los Servicios del Ecosistema (N)

\begin{tabular}{|l|l|l|l|l|l|}
\hline 6 & 14 & 1307 & 984 & 93,36 & 163,64 \\
\hline 13 & 9 & 1103 & 729 & 122,56 & 203,56 \\
\hline 14 & 5 & 397 & 315 & 79,40 & 142,40 \\
\hline 15 & 14 & 1020 & 789 & 72,86 & 129,21 \\
\hline & 42 & 3827 & 2817 & 368,17 & 638,81 \\
\hline
\end{tabular}

Asignación eficiente. Contribución Económica Neta (E)

\begin{tabular}{|l|l|l|l|l|l|}
\hline 7 & 8 & 971 & 698 & 121,38 & 208,63 \\
\hline 8 & 75 & 17778 & 12088 & 237,04 & 398,21 \\
\hline 9 & 49 & 4569 & 3259 & 93,24 & 159,76 \\
\hline 11 & 51 & 4402 & 3203 & 86,31 & 149,12 \\
\hline 12 & 22 & 2083 & 1497 & 94,68 & 162,73 \\
\hline & 205 & 29803 & 20745 & 632,66 & 1078,44 \\
\hline
\end{tabular}

Fuente: Elaboración propia.

En la Tabla 5 se observa que el impacto del Capital Social/Contribución comunitaria (elemento del bienestar) es considerablemente superior $(2492,94)$ al Capital Natural / Contribución a los Servicios del Ecosistema (elemento medioambiental y sostenible) y a la Contribución Económica Neta (elemento económico) cuyos valores 
son de 638,81 y 1078,44 respectivamente. Por tanto, en el usuario @desdelamoncloa el elemento del bienestar tiene mayor impacto que el medioambiental y económico.

\section{Conclusiones y discusión}

En este trabajo se ha mostrado cómo la red social de microblogging Twitter es una herramienta que desde el punto de vista del marketing social se puede utilizar para obtener información sobre el interés de los usuarios en los ODS y contribuir en la tomar decisiones en la elaboración e implantación de las políticas públicas, compartiendo con Rodríguez y Ureña (2011) que Twitter es un medio que hace más cercanas las demandas y opiniones de los ciudadanos a sus políticos; pero también es una vía por la cual los políticos pueden influir en la opinión de los ciudadanos, concienciándolos sobre la idoneidad de implantar de una forma más eficientes políticas de bienestar.

En los resultados del análisis cualitativo, concretamente en el análisis del contexto del vocablo «bienestar», se observa que este término aparece con otras palabras como «Estado» o con los hashtags \#40AñosdeEstadoSocial o \#SomosEspaña, lo que vienen a indicar la preocupación del gobierno por los valores que representa España y por el bienestar de los ciudadanos. Esta cuestión se analiza en multitud de estudios que recogen la necesidad de dicha responsabilidad, sobre todo desde que surgieran el enfoque de la economía institucional (Vergara y Ortiz, 2016). Es también de destacar que hay un número considerable de referencias en la que la finalidad es de conversación y acción al realizar preguntas sobre los ODS y animar a vinculares a través de las redes sociales (Saxton \& Waters, 2014).

En los resultados del análisis cuantitativo se muestra que los mensajes que hablan del objetivo 8, es decir, «trabajo decente y crecimiento económico» han sido los que han tenido un mayor impacto, sin embargo, no son los más publicados. Lo contrario sucede con los mensajes que hablan del objetivo 10, «reducción de las desigualdades». Por otra parte, los mensajes con menos reacciones son los que hablan del ODS 15 y 11. Estos resultados contradicen para el caso de @desdelamoncloa los obtenidos por los estudios de Menéndez, Saura y Álvarez (2018), Can y Atlatas (2017) y Angulo (2010), en los que se concluye que el «medio ambiente» es uno de los temas que más preocupan, así como la «salud y el bienestar».

Asimismo, a partir del análisis de @desdelamoncloa sobre el bienestar y los ODS se ha comprobado que a pesar de que el cumplimiento de la Agenda 2030 y los 17 ODS son esenciales para el futuro del país, no se suelen publicar mensajes que traten directamente sobre ellos.

En relación a las implicaciones políticas del análisis de los objetivos según los tres elementos (Bienestar, Economía y Sostenibilidad), se ha podido constatar que el impacto ha sido mayor en los objetivos enfocados al bienestar y a la economía que a la sostenibilidad. Esto nos puede llevar a pensar que hay poca concienciación del deterioro del medio ambiente y que sería necesario sensibilizar a la población española sobre el problema medioambiental para implicarlos en el cumplimiento de los ODS.

En esta línea, Liu et al. (2014) propusieron la creación de un observatorio ciudadano para potenciar la concienciación ciudadana sobre el deterioro del medioambiente y sostienen una gobernanza ambiental basada en la comunidad. Creemos que 
esta propuesta puede ser interesante, pero discrepamos en que sea el único lugar virtual para recopilar y compartir datos. Estamos de acuerdo que el problema del medio ambiente es un problema global, pero creemos que la acción política se debe realizar tanto desde una perspectiva global como de forma particular por cada uno de los países (glocal). La concienciación ciudadana medioambiental de cada país es diferente, pues existen países como Islandia y Noruega donde sus ciudadanos tienen una alta concienciación sobre el medioambiente, por tanto, su acción política sería diferente a la que se tendría que hacer en España.

En definitiva, este estudio ha mostrado que Twitter proporciona información por un lado sobre cuáles son los ODS que el usuario de la cuenta oficial del gobierno en España considera en mayor medida, y, por otro lado, los Objetivos de Desarrollo Sostenible que más preocupan a sus usuarios. Además, revela con cuáles está relacionado el tema del bienestar y aporta información útil para saber la línea a seguir para la concienciación a los ciudadanos. Sería conveniente seguir investigando en esta línea para ayudar a las organizaciones a avanzar en la gestión del marketing social por medio de las redes sociales digitales ya que suponen una gran oportunidad para que los gobiernos comuniquen sus proyectos y creen relaciones a largo plazo con la comunidad en aras a convertirlos en colaboradores.

\section{Referencias}

Aboelmaged, M. (2018). Predicting the success of Twitter in healthcare: A synthesis of perceived quality, usefulness and flow experience by healthcare professionals. Online Information Review, 42(6), 898-922. https://doi.org/10.1108/OIR-01-2017-0018

Agencia Española de Cooperación Internacional para el Desarrollo (AECID). (2019). La Agenda 2030. El reto de los Objetivos de Desarrollo Sostenible, 15 de mayo del 2019. Recuperado de https://bit.ly/29ZqPSq

Andreasen, A. (1994). Social Marketing: Its Definition and Domain. Journal of Public Policy Eु Marketing, 13(1), 108-114. https://doi.org/10.1177\%2F074391569401300109

Angulo, N. (2010). Pobreza, medio ambiente y desarrollo sostenible. Nómadas, Revista Crítica de Ciencias Sociales y Jurídicas, 26(2), 1-11.

Asheim, G. (2010). The relationship between welfare measures and indicators of sustainable development. En T. Aronsson \& K.-G. Löfgren (Eds.), Handbook of Environmental Accounting (pp. 237-256). England: Edward Elgar Publishing LTD. https://doi. org/10.4337/9781849805704

UN General Assembly (2015). Transforming our world: the 2030 agenda for sustainable development. New York, Estados Unidos: United Nations.

Bain, J., \& Chaban, N. (2017). An emerging EU strategic narrative? Twitter communication during the EU's sustainable energy week. Comparative European Politics, 15(1), 135-155. https:// doi.org/10.1057/cep.2016.17

Bernhardt, J., Mays, D., \& Hall, A. (2012). Social marketing at the right place and right time with new media. Journal of Social Marketing, 2(2), 130-137. https://doi.org/10.1108/20426761211243964

Chisholm, E., \& O’Sullivan, K. (2017). Using Twitter to Explore (un)Healthy Housing: Learning from the \#Characterbuildings Campaign in New Zealand. Int. J. Environ. Res. Public Health, 14(11), 1424. https://dx.doi.org/10.3390\%2Fijerph14111424

Blanchflower, D. G., \& Oswald, A. J. (2011). International happiness: A new view on the measure of performance. Academy of Management Perspectives, 25(1), 6-22. https://doi.org/10.5465/ amp.25.1.6

Chen, J., Goldstein, M., Asch, S., Mackey, L., \& Altman, R. (2017). Using social media to monitor 
mental health discussions-evidence from Twitter. Journal of the American Medical Informatics Association, 24(3), 496-502. https://doi.org/10.1093/jamia/ocw133

Chua, H., Allem, P., Unger, J., Cruz, T., Akbarpour, M., \& Kirkpatrick, M. (2019). Strategies to find audience segments on Twitter for e-cigarette education campaigns. Addictive Behaviors, 91, 222-226. https://doi.org/10.1016/j.addbeh.2018.11.015

Colditz, J., Welling, J., Smith, N., James, A., \& Primack, B. (2019). World Vaping Day: Contextualizing vaping culture in online social media. Journal of Mixed Methods Research, 13(2), 1-20. https://doi.org/10.1177/1558689817702753

Costanza, R., Daly, L., Fioramonti, L., Giovannini, E., Kubiszewski, I., Mortensen, L. F., \& Wilkinson, R. (2016). Modelling and measuring sustainable wellbeing in connection with the UN Sustainable Development Goals. Ecological Economics, 130, 350-355. http://dx. doi.org/10.1016/j.ecolecon.2016.07.009

Costanza, R., McGlade, J., Lovins, H., \& Kubiszewski, I. (2014). An overarching goal for the UN sustainable development goals. Solutions, 5(4), 13-16.

Coulthard, S., Johnson, D., \& McGregor, J. A. (2011). Poverty, sustainability and human wellbeing: a social wellbeing approach to the global fisheries crisis. Global Environmental Change, 21(2),. 453-463. https://doi.org/10.1016/j.gloenvcha.2011.01.003

Di Tella, R. D., MacCulloch, R. J., \& Oswald, A. J. (2003). The macroeconomics of happiness. Review of Economics and Statistics, 85(4), 809-827. https://doi.org/10.1162/003465303772815745

Di Tella, R., Haisken-De New, J., \& MacCulloch, R. (2010). Happiness adaptation to income and to status in an individual panel. Journal of Economic Behavior \& Organization, 76(3), 834-852. https://dx.doi.org/10.1016/j.jebo.2010.09.016

Di Tella, R., MacCulloch, R. J., \& Oswald, A. J. (2001). Preferences over inflation and unemployment: Evidence from surveys of happiness. American economic review, 91(1), 335-341. https:// dx.doi.org/10.1257/aer.91.1.335

Diener, E., Lucas, R. E., \& Scollon, C. N. (2009). Beyond the hedonic treadmill: Revising the adaptation theory of well-being. American Psychologist, 61(4), 305-314. https://doi. org/10.1037/0003-066X.61.4.305

DiMaria, C. (2019). Sustainability, welfare and efficiency of nations. Quality $\mathcal{E}$ Quantity, 53(3), 1141-1163. https://doi.org/10.1007/s11135-018-0809-3

Domegan, C., Kollins, K., Stead, M., McHugh, P., \& Hughes, M. (2013). Value co-creation in social marketing: functional or fanciful. Journal of Social Marketing, 3(3), 239-256. https://doi. org/10.1108/JSOCM-03-2013-0020

Dooley, J., Jones, S., \& Iverson, D. (2012). Web 2.0: an assessment of social marketing principles. Journal of Social Marketing, 2(3), 207-221. https://doi.org/10.1108/20426761211265195

Duarte, T., \& Jiménez, R. E. (2007). Aproximación a la teoría del bienestar. Scientia et Technica, 13(37), 305-310. http://dx.doi.org/10.22517/23447214.4107

Durana, D., Artenea, A., Gogana, L., \& Durana, V. (2015). The Objectives of Sustainable Development - Ways to Achieve Welfare. Procedia Economics and Finance, 26, 812-817. https://doi. org/10.1016/S2212-5671(15)00852-7

Easterlin, R. A., McVey, L. A., Switek, M., Sawangfa, O., \& Zweig, J. S. (2010). The happiness-income paradox revisited. Proceedings of the National Academy of Sciences, 107(52), 22463-22468. https://doi.org/10.1073/pnas.1015962107

Fisher, C. (2010). Happiness at work. International journal of management reviews, 12(4), 384-412. https:// doi.org/10.1111/j.1468-2370.2009.00270.x

Fownes, J., Yu, C., \& Margolin, D. (2018). Twitter and climate change. Sociology Compass, 12(6), 1-12. https://doi.org/10.1111/soc4.12587

Frey, B. S., \& Stutzer, A. (2018). Economics of happiness. Switzerland: Springer International Publishing. https://doi.org/10.1007/978-3-319-75807-7

Froding, K., Eriksson, C., Elander, I., \& Geidne, J. (2007). Partnership for healthy neighbourhoods: City Networking in Multilevel Context. European Journal of Public Health 15(4), 317- 
331. https://doi.org/10.1177\%2F0969776408095108

Gallopín, G. (2003). Sostenibilidad y desarrollo sostenible: un enfoque sistémico. Santiago de Chile: CEPAL.

Gerdtham, U. G., \& Johannesson, M. (2001). The relationship between happiness, health, and socio-economic factors: results based on Swedish microdata. The Journal of Socio-Economics, 30(6), 553-557. https://doi.org/10.1016/S1053-5357(01)00118-4

Golbeck, J., \& Hansen, D. (2014). A method for computing political preference among Twitter followers. Social Networks, 36, 177-184. https://doi.org/10.1016/j.socnet.2013.07.004

Griggs, D., Stafford-Smith, M., Gaffney, O., Rockström, J., Öhman, M. C., Shyamsundar, P., ... \& Noble, I. (2013). Policy: Sustainable development goals for people and planet. Nature, 495(7441), 305-307. https://doi.org/10.1038/495305a

Hestres, L. (2014). Preaching to the choir: internet-mediated advocacy, issue public mobilization, and climate change. New Media $\mathcal{F}$ Society, 16(2), 323-339. https://doi. org/10.1177/1461444813480361

IAB Spain \& Elogia (2018). VIII Estudio anual de redes sociales 2018. Recuperado de https://bit.ly/ 2J95UhE

Kotler, P. (2011). Reinventing marketing to manage the environmental imperative. Journal of Marketing, 75(4), 132-135. https://doi.org/10.1509/jmkg.75.4.132

Kotler, P., \& Levy, S. (1969a). Broadening the Concept of Marketing. Journal of Marketing, 33(1), 10 15. https://doi.org/10.1177\%2F002224296903300103

Kotler, P., \& Levy, S. (1969b). A New Form of Marketing Myopia: Rejoinder to Professor Luck. Journal of Marketing, 33(3), 55-57. https://doi.org/10.2307/1248483

Krippendorff, K. (1990). Metodología de análisis de contenido. Teoría y práctica. Barcelona, España: Paidós Comunicación.

Liu, H. Y., Kobernus, M., Broday, D., \& Bartonova, A. (2014). A conceptual approach to a citizens' observatory-supporting community-based environmental governance. Environmental Health, 13(1), 107. https://doi.org/10.1186/1476-069X-13-107

Luttmer, E. F. (2005). Neighbors as negatives: Relative earnings and well-being. The Quarterly journal of economics, 120(3), 963-1002. https://doi.org/10.1093/qje/120.3.963

McGregor, S., \& Mourao, R. (2016). Talking Politics on Twitter: Gender, Elections, and Social Networks. Social Media + Society, 2(3), 1-14. https://doi.org/10.1177\%2F2056305116664218

Menéndez, A.; Saura, J., \& Álvarez, C. (2018). Understanding \#WorldEnvironmentDay User Opinions in Twitter: A Topic-Based Sentiment Analysis Approach. International Journal of Environmental Research and Public Health, 15(11), 2537. https://doi.org/10.3390/ijerph15112537

Mikulcic, H., Klemes, J., \& Duic, N. (2016). Shaping sustainable development to support human welfare. Clean Technologies and Enviromental Policy, 18(6), 1633-1639. https://doi.org/10.1007/ s10098-016-1269-x

Moll de Alba, E. (2015). Análisis comparativo de la utilización de Twitter como canal de comunicación para las principales editoriales estadounidenses y españolas (tesis doctoral). Universidad Autónoma de Barcelona, España.

Momete, D. (2017). Rational Development as a Sustainable Progress Welfare Vector: A Cross-Country Analysis. Sustainable Development, 25(3), 189-199. https://doi.org/10.1002/ sd. 1645

Neuendorf, K. (2002). The content analysis guidebook. Estados Unidos: Sage.

Pearce, W.; Holmberg, K.; Hellsten, I. y Nerlich, B. (2014). Climate change on Twitter: topics, communities and conversations about the 2013 IPCC Working Group 1 report. PLoS One, 9(4), 1-12. https://doi.org/10.1371/journal.pone.0094785 
Pershad, Y., Hangge, P., Albadawi, H., \& Oklu, R. (2018). Social Medicine: Twitter in Healthcare. Journal of Clinical Medicine, 7(6), 1-9. https://doi.org/10.3390/jcm7060121

Ravina Ripoll, R., Villena Manzanares, F., \& Gutiérrez Montoya, G. A. (2017). Una aproximación teórica para mejorar los resultados de innovación en las empresas desde la perspectiva del "Happiness Management". Retos. Revista de Ciencias de la Administración y Economía, 7(14), 113-129. https://doi.org/10.17163/ret.n14.2017.06

Rodrigo-Cano, D., Picó, M.J., \& Dimuro, G. (2019). Los Objetivos de Desarrollo Sostenible como marco para la acción y la intervención social y ambiental. Retos. Revista de Ciencias de la Administración y Economía, 9(18), 25-36. https://doi.org/10.17163/ret.n17.2019.02

Rodríguez, R., \& Ureña, D. (2011). Diez razones para el uso de Twitter como herramienta en la comunicación política y electoral. Comunicación y pluralismo, (10), 89-116.

Rogers, D. S., Duraiappah, A. K., Antons, D. C., Munoz, P., Bai, X., Fragkias, M., \& Gutscher, H. (2012). A vision for human well-being: transition to social sustainability. Current Opinion in Environmental Sustainability, 4(1), 61-73. https://doi.org/10.1016/j.cosust.2012.01.013

Rojas, M. (2009). Economía de la felicidad: hallazgos relevantes respecto al ingreso y el bienestar. El trimestre económico, 76(303), 537-573. https://www.jstor.org/stable/20857218

Saxton, G., \& Waters, R. (2014). What do Stakeholders Like on Facebook? Examining Public Reactions to Nonprofit Organizations' Informational, Promotional, and Community-Building Messages. Journal of Public Relations Research, 26(3), 280-299. https://doi.org/10.1080/106 2726X.2014.908721

Sammarco, P. (2018). V informe del uso de las redes sociales en España, Social Media Family (SMF), 20 de febrero del 2019. Recuperado de https://bit.ly/2BR2FHL

Stiglitz, J., Sen, A., \& Fitoussi, J. P. (2008). Informe de la Comisión sobre la Medición del Desarrollo Económico y del Progreso Social. Recuperado de https://bit.ly/2v0ZJmy

Urbina, M., Gutiérrez, L., Ermani, R., Lozano, R., \& Finkelman, J. (2017). La transición de los Objetivos de Desarrollo del Milenio a los Objetivos de Desarrollo Sostenible desde la perspectiva de los determinantes sociales de la salud y la equidad en salud. Gac Med Mex, 153(6), 697-730.

Vergara, C., \& Ortiz, D. (2016). Desarrollo sostenible: enfoques desde las ciencias económicas. Apuntes del CENES, 35(62), 15-52. https://doi.org/10.19053/22565779.4240

Zhang, Z., \& Ahmed, W. (2019). A comparison of information sharing behaviors across 379 health conditions on Twitter. International Journal of Public Health, 64(3), 431-440. https://doi. org/10.1007/s00038-018-1192-5 